\title{
Machine learning and intelligence science: IScIDE (C)
}

\author{
(c) Higher Education Press and Springer-Verlag Berlin Heidelberg 2012
}

Two closely related series of efforts have been organized in the past three years, towards a promotion of Sino-foreigninterchange on interdisciplinary areas of intelligence science, information science, and intelligent data engineering, and also in memory of thirty year anniversary of the Zhongguancun Golden Triangle ISIS Forum founded and propelled by the late senior academicians Prof. T. Chang of Tsinghua University and his collaborator, Prof. M. T. Cheng of Peking University, see the second paragraph of Ref. [1].

One is a serial annual workshops on Intelligence Science and Intelligent Data Engineering (IScIDE) as a collective venue for introducing world frontier researchers to China and for introducing researchers from Chinese major universities and research institutions to international communities. Already, IScIDE2010 and IScIDE2011 were held on June 3-5, 2010 in Harbin and on October 23-25, 2011 in Xi'an, respectively. In addition to having keynote speakers, oral and posters presentations as typically in standard international conferences, one salient feature of the workshops is a single track of oral sessions, which consists of not only strictly selected oral presentations and poster-spotlights but also particularly arranged invited talks by core researchers from Chinese top universities and by their corresponding foreign researchers. For IScIDE2010 and IScIDE2011, there have been in total such invited presentations with 18 from mainland and 15 outside mainland.

Another series of efforts were originally scheduled to publish three special issues on Frontiers of Electrical and Electronic Engineering in China. The first one is named Emerging themes on information theory and Bayesian approach (Vol. 5, No. 3, 2010, shortly V5N3) which consists of nine invited papers from international well-known research pioneers and Chinese senior scientists. The other two special issues come from speakers of IScIDE2010, namely, Machine learning and intelligence science: Sino-foreign-interchange workshop IScIDE2010 (A) (Vol. 6, No. 1, 2011, shortly V6N1) and IScIDE2010 (B) (Vol. 6, No. 2, 2011, shortly V6N2). V6N1 consists of 14 invited papers, roughly with a half from top universities in mainland China and the other half from universities outside of mainland China. V6N2 consists of 19 papers, with a majority coming from major universities in China and the first authors of all these papers are Ph.D candidates, which provides a typical window for international communities to observe the researches by Ph.D candidates in China.

After publication of the above three special issues, there were feedbacks that the coverage of V6N1 missed some representative professors and some top universities in mainland China, and a supplementary issue is expected. Thus, we schedule this special issue as a continuation of V6N1, which is named IScIDE (C) since the invited authors mostly come from the invited speakers of IScIDE2010 and IScIDE2011. Keeping the V6N1 style, it is scheduled as the first issue of a year (Vol. 7, No. 1, 2012, shortly V7N1) and the first author of each paper is invited. Originally, authors of 18 papers have accepted invitation. However, from initialization to publication, there had been less than a half year for a schedule of review, revision, and production. Unfortunately, five papers (though three in manuscript revision already) could not reach the editorial office before the final deadline of production. We are sorry about such a rigid time schedule and express our thanks to the efforts made by these authors. Anyway, these special issues may still serve as a representative window for observing the achievements of professors in China. Actually, these authors

Received January 19, 2012

Lei XU

Department of Computer Science and Engineering, The Chinese University of Hong Kong, Hong Kong, China

E-mail: lxu@cse.cuhk.edu.hk

Yanda LI

Department of Automation, Tsinghua University, Beijing 100084, China

E-mail: daulyd@tsinghua.edu.cn 
come from more than 20 major universities in China (including the topmost nine universities in the mainland). Among them, eleven professors are recipients of Chinese NSF Distinguished Young Scholars, while others are also recipients of various levels of academic honors.

This V7N1 consists of 13 papers that are bisected. The first half covers two parts of studies that are actually beyond but closely related to machine learning and intelligence science (ML\&IS). One part consists of GPU computing, evolutionary computing, and neurocomputing, which are topics that have not been involved in V6N1 and V6N2. The other part covers three application areas that were partially involved by Refs. [2-4] in V6N2. Three papers in this V7N1 not only address new applications, but also provide brief overviews. The second half covers two essential fields of ML\&IS. One is visual-audio perception and modeling, also partially involved by Ref. [5] in V6N1, Ref. [6] in V5N3, and Refs. $[7,8]$ in V6N2, while new topics will addressed by three papers in this V7N1. The other essential field is learning algorithms and model selection, addressed by four papers that supplement to the studies on the related topics in V6N1 and V6N2.

We start with the first field, i.e., GPU computing, evolutionary computing, and neurocomputing. The first article is written by Dr. Kun Zhou from Zhejiang University, a professor of Education Ministry Cheung Kong Scholar and one of Chinese NSF Distinguished Young Scholars. Prof. Zhou is among a few leading mainland researchers in computer graphics and GPU computing, and also known internationally as one of the world's top 35 young innovators by MIT Technology Review in 2011. This paper introduces his recent achievements that make GPU software development much easier, including a user friendly high-level programming language BSGP for generalpurpose computation on the GPU, a BSGP's debugging system based on the GPU interrupt, and some widely-used spatial hierarchies for high-performance visual computing applications. Actually, many ML\&IS algorithms can be significantly accelerated by making use of GPU's parallel processing power. Also, encountered widely in many ML\&IS studies are optimization problems too. The second paper provides a survey on evolutionary computation that tackles optimization problems by emulating biologic evolution and organizational behaviors, written by a team of Sun Yat-sen University led by Prof. Jun Zhang, a recipient of Chinese NSF Distinguished Young Scholars in 2011. The survey is featured by classifying existing adaptation schemes into categories. It further suggests to estimate the evolutionary state information for designing effective adaptation schemes, exemplified by two representative algorithms. The third article is written by authors from Fudan University, the invited first author is Dr. Wenlian $\mathrm{Lu}$, a recipient of Chinese National Excellent Doctoral Dissertation Award. This paper provides a review of their studies on a typical neurocomputing system for learning dynamical process, called recurrent neural networks. The Filippov solution is employed to define the system solution, and the theory of viability provides a tool to study the existence and uniqueness of the solution, and the Lyapunov approach is used to investigate the global stability and synchronization.

The second part of the first half covers three representative application tasks. The first one is radar automatic target recognition (RATR) based on high resolution range profile (HRRP). The National Laboratory of Radar Signal Processing, Xidian University, is one of the major radar signal processing research institutes that have contributed considerably to the HRRP-based RATR literature. The first paper is written by a team led by Academician Prof. Zheng Bao and the Laboratory Director Prof. Hongwei Liu, which provides an overview on their recent studies and also presents a new noise robust recognition algorithm. The second paper deals with the task for controlling a flexible air-breathing hypersonic vehicle and presents a polytopic linear parameter varying model with a non-fragile $H_{2}$ gain-scheduled control. The paper is written by the team of Prof. Changyin Sun from Southeast University, also a recipient of Chinese NSF Distinguished Young Scholars in 2011. The third paper addresses structure-based drug design and NMR structural biology, written by Prof. Lincong Wang and colleagues from Jilin University, giving not only an introduction on algorithmic challenges but also two novel docking algorithms. In this area, Prof. Wang is one of representative mainland researchers, e.g., published papers on PNAS, J. Biol. Chem., J. Mol. Biol., J. Am. Chem. Soc., and received Best Paper Awards on CSB 2006 and CSB 2003.

Next, we move to the second half of this special issue and introduce three articles on visual-audio perception and modeling. The first article is written by Prof. Fang Fang and co-author from Peking University, which provides a review on their recent fMRI and psychophysical findings, indicating that the human early visual cortex not only performs local feature analyses, but also contributes significantly to high-level visual computation with assistance of attention-enabled cortical feedback. Moreover, a biologically plausible attention model is proposed to predict human scanpaths on natural images. Prof. Fang is a recipient of Chinese NSF Distinguished Young Scholars, and one of a few leading mainland researchers who have published papers in international top journals, e.g., Nature Neuroscience, Neuron, PNAS. The second article is written by authors lead by Prof. Alan Yuille from UCLA, an IEEE Fellow and a recipient of Marr Prize. Tackling the difficulty of extremely demanding computation for probabilistic models of vision, this paper shows that computationally tractable approximations can be made by placing bounds on the 
normalization term of probability distribution. For certain choices of loss functions, many of the standard maxmargin criteria used in support vector machines are obtained, and thus many machine learning methods can be obtained as approximations to existing typical probabilistic methods. The third article proposes a computational auditory model for assessment of speech intelligibility, written by $\mathrm{Wu}$ and Chen from Peking University, where Prof. Xihong Wu is the director of Speech and Hearing Research Center, Key Laboratory of Machine Perception (Ministry of Education). In addition to evaluating speech intelligibility merely by energetic masking, the proposed model also characterizes the difficulty of extracting harmonic feature of the target signal. This model predicts highly consistent with experiments, and outperforms the existing models.

The last but not least, the last four papers supplement the streams of studies already introduced in V6N1 and V6N2. These streams all share a feature that many Chinese researchers have not only put efforts on them but also made some unique and influential contributions. One of them is describing data samples with reduced dimensionality, i.e., one of the two focused streams in V6N1, for which readers are referred to an outline in Ref. [1] and a previous survey in Ref. [9], to the papers [10-13] in V6N1 and the papers [14-16] in V6N2. In this V7N1, the paper by Gao and Wang not only supplements with a brief overview on dimensionality reduction algorithms but also presents the contributions by the team of Prof. Xinbo Gao from Xidian University, another recipient of Chinese NSF Distinguished Young Scholars in 2011. They introduce two dimensionality reduction algorithms based on the Gaussian process latent variable model and further use transfer learning techniques to deal with samples of not independent identically distributed. Next, the paper by authors headed by Prof. Jufu Feng from Peking University works on the Breiman's margin explanation of the AdaBoost algorithm. A new margin termed Emargin is proposed as indicator of generalization, by which a new sharper bound is obtained for generalization error of voting classifiers. Experiments show that AdaBoost provides a better Emargin than arc-gv in most cases. Also, this paper falls in one of the two focused streams of $\mathrm{V} 6 \mathrm{~N} 1$, that is, integrating multi-sources of partial information for a better performance [1]. Other studies are referred to Refs. [17-19] in V6N1 and a previous survey [20], as well as to Sect. 4.4 of Ref. [21] in V5N3 and Sect. 3.3.2 of the last paper in this V7N1.

Moreover, V6N1 and V6N2 also cover other two streams on which many Chinese researchers focus on. One is kernel based learning, see Ref. [12] in V6N1 and Ref. [22] in V6N2, while the other is making clustering analyses or learning Gaussian mixture with automatic model selection, see Refs. [22,23] in V6N2 and Sect. 3.1 of Ref. [21] in V5N3, as well as Sect. 2 of the last paper in this V7N1. The paper by Prof. Jianhuang Lai and co-author from Sun Yat-sen University is actually in both the two streams, which presents two kernel based competitive learning clustering algorithms. One is derived from the viewpoint of kernel clustering, and the other is based on the graph method with multi-prototype representation introduced to characterize clusters of irregular shapes. Finally, the last paper in this V7N1 serves as a supplementary of Ref. [21] in V5N3, which summarizes essential topics of the BYY harmony learning, including current status of efforts made, challenging and demanding issues about the BYY system design, the best harmony learning principle, techniques for implementation, and typical inner dependence structures in the BYY system, as well as some gene analysis applications.

Even with this V7N1, the coverage of this four-issue series may still miss some representative professors and universities in China. If not impossible, it is extremely difficult to reach a completed coverage. Anyway, it is time to conclude this series. Finally, we would express our sincere thanks to all the authors of the four special issues for their valuable contributions.

Acknowledgements This work by the guest editor was partially supported by National Key Basic Research and Development Program under Grant No. 2009CB825404. The guest editor is a member of the Steering Committee for Information Sciences under CUHK focused investments scheme, to which this work was partially dedicated.

Lei XU

Guest Editor of the Special Issue

Yanda LI

Editor-in-Chief of Frontiers of Electrical and Electronic Engineering

\section{References}

1. Xu L, Li Y D. Machine learning and intelligence science: Sino-foreign interchange workshop IScIDE2010 (A). Frontiers of Electrical and Electronic Engineering in China, 2011, 6(1): 1-5 
2. Wang P H, Shi L, Du L, Liu H W, Xu L, Bao Z. Radar HRRP statistical recognition with temporal factor analysis by automatic Bayesian Ying-Yang harmony learning. Frontiers of Electrical and Electronic Engineering in China, 2011, 6(2): 300-317

3. Mu C X, Sun C Y. Data-based intelligent modeling and control for nonlinear systems. Frontiers of Electrical and Electronic Engineering in China, 2011, 6(2): 291-299

4. Ye C, Liu L X, Wang X, Zhang X G. Observations on potential novel transcripts from RNA-Seq data. Frontiers of Electrical and Electronic Engineering in China, 2011, 6(2): 275-282

5. Hu D W, Zhou Z T, Wang Z Z. Processing real-world imagery with FACADE-based approaches. Frontiers of Electrical and Electronic Engineering in China, 2011, 6(1): 120-136

6. Yuille A. An information theory perspective on computational vision. Frontiers of Electrical and Electronic Engineering in China, 2010, 5(3): 329-346

7. Zhou L, Hu D W, Zhou Z T, Zhuang Z W. Natural scene recognition using weighted histograms of gradient orientation descriptor. Frontiers of Electrical and Electronic Engineering in China, 2011, 6(2): 318-327

8. Pang Z H, Tu S K, Su D, Wu X H, Xu L. Discriminative training of GMM-HMM acoustic model by RPCL learning. Frontiers of Electrical and Electronic Engineering in China, 2011, 6(2): 283-290

9. Xu L. Independent subspaces. In: Ramón J, Dopico R, Dorado J, Pazos A, eds. Encyclopedia of Artificial Intelligence. Hershey, PA: IGI Global, 2008, 903-912

10. Yin H. Advances in adaptive nonlinear manifolds and dimensionality reduction. Frontiers of Electrical and Electronic Engineering in China, 2011, 6(1): 72-85

11. He X F, Lin B B. Tangent space learning and generalization. Frontiers of Electrical and Electronic Engineering in China, 2011, 6(1): $27-42$

12. Yang J. Kernel feature extraction methods observed from the viewpoint of generating-kernels. Frontiers of Electrical and Electronic Engineering in China, 2011, 6(1): 43-55

13. Xu L. Codimensional matrix pairing perspective of BYY harmony learning: Hierarchy of bilinear systems, joint decomposition of data-covariance, and applications of network biology. Frontiers of Electrical and Electronic Engineering in China, 2011, 6(1): 86-119

14. Tu S K, Xu L. Parameterizations make different model selections: Empirical findings from factor analysis. Frontiers of Electrical and Electronic Engineering in China, 2011, 6(2): 256-274

15. Tu S K, Xu L. An investigation of several typical model selection criteria for detecting the number of signals. Frontiers of Electrical and Electronic Engineering in China, 2011, 6(2): 245-255

16. Zhang L J, Chen Z G, Zheng M, He X F. Robust non-negative matrix factorization. Frontiers of Electrical and Electronic Engineering in China, 2011, 6(2): 192-200

17. Zhou Z H. When semi-supervised learning meets ensemble learning. Frontiers of Electrical and Electronic Engineering in China, 2011, 6(1): 6-16

18. Zhang C S, Wang F. Graph-based semi-supervised learning. Frontiers of Electrical and Electronic Engineering in China, 2011, 6(1): $17-26$

19. Lu B L, Wang X L, Yang Y, Zhao H. Learning from imbalanced data sets with a Min-Max modular support vector machine. Frontiers of Electrical and Electronic Engineering in China, 2011, 6(1): 56-71

20. Xu L, Amari S. Combining classifiers and learning mixture-of-experts. In: Dopico J R R, Dorado J, Pazos A, eds. Encyclopedia of Artificial Intelligence. 2009, 318-326

21. Xu L. Bayesian Ying-Yang system, best harmony learning, and five action circling. Frontiers of Electrical and Electronic Engineering in China, 2010, 5(3): 281-328

22. Liao L, Zhang Y N. MRI image segmentation based on fast kernel clustering analysis. Frontiers of Electrical and Electronic Engineering in China, 2011, 6(2): 363-373

23. Shi L, Tu S K, Xu L. Learning Gaussian mixture with automatic model selection: A comparative study on three Bayesian related approaches. Frontiers of Electrical and Electronic Engineering in China, 2011, 6(2): 215-244 